\section{Discussion}

The present series is too small to allow more than cautious optimism about the results of immunotherapy, and in contrast to the St. Bartholomew's group (Crowther et al., 1973) the duration of first bone marrow remission did not seem to be obviously greater as compared with patients receiving maintenance chemotherapy alone. Nevertheless, immunotherapy has the great advantage that it has few side effects.

The high rate of reinduction achieved is very encouraging. The best reported figures for reinduction rates are around $30-40 \%$ (Wiernik, et al., 1970; Crowther et al., 1973), though in our hands the reinduction rate was previously not more than $12 \%$ (Bailey et al., 1971). Moreover, third and fourth remissions are extremely rare. It is not clear whether the high reinduction rate in our patients treated with immunotherapy was a positive result of immunotherapy or attributable to the absence of maintenance chemotherapy and the avoidance of drug resistance. The latter explanation seems perhaps less likely in view of the fact that only one out of five patients who went into remission in the fourth and fifth M.R.C. trials on acute myeloid leukaemia and who for various reasons received no further treatment achieved a remission after subsequent relapse (Galton, 1973). Haddow and Alexander (1964) reported that the effectiveness of a single dose of $2,000 \mathrm{r}$ of $x$-rays in retarding the growth of primary rat fibrosarcoma can be enhanced by an autograft of the tumour. One patient with acute lymphoblastic leukaemia treated with immunotherapy at the Royal Manchester Children's Hospital, Pendlebury (Morris Jones, 1973), was put back on to the same maintenance chemotherapy as he had received before immunotherapy. The same dose of drugs produced marked marrow hypoplasia, suggesting an increased sensitivity of the normal marrow elements to chemotherapy.

The ease of reinduction might also be attributed to the relatively early diagnosis of relapse in our series. In most patients relapse was based on the results of bone marrow examination which we performed monthly, and only occasionally was there any change in the peripheral blood counts. Only the one patient who eventually died and a further patient after his second relapse showed clinical signs of relapse.

Centres where bone marrow examination is performed less often and which depend more on clinical and peripheral blood evidence of relapse may find greater difficulty in reinducing a remission. Though the apparent duration of first remission may be greater overall survival would then be shorter.

We are deeply indebted to our many colleagues in the departmen's of haematology and medical genetics for their clinical and technical help. Our thanks are due particularly to Miss G. Marriott and Miss M. Enright for their nursing and technical help, to Dr. D. Dawson at Crumosall Hospital for allowing us to include in the trial two patients under his care, to Dr. I. Garrett, Dr. P. Massey, and Miss Souter and her staff for their help in the preparation and irradiation of the leukaemia cells, and to Miss Scholes at Manchester Royal Infirmary for her technical help. We are graieful to the Leukaemia Research Fund, the Medical Research Council, the United Manchester Hospitals Board of Governors, the research grants committee, and G. D. Searle and Co., for financial help and to the department of medical illustration, Manchester Royal Infirmary, for their help with the illustration.

ADDENDUM.-The latest data on patients receiving immunotherapy are as follows. Case 1 relapsed at 95 weeks and has since achieved a fifth remission (current overall survival 107 weeks). Case 14 died without achieving a further remission after a total of 72 weeks. Cases 19 and 29 are still in their first remission (duration 73 and 55 weeks respectively; current overall survival 78 and 60 weeks respectively). Case 21 remains in his second remission (duration 42 weeks; current overall survival 76 weeks). Case 26 relapsed for a second time at 64 weeks but has achieved a third remission (current overall survival 71 weeks).

\section{References}

Bailey, C. C., et al. (1971). Lancet, 1, 1268.

Buckner, D., Graw, R. G., Eisel, R. J., Henderson, E. S., and Perry, S. (1969). Blood, 33, 353.

Crowther, D., et al. (1973). British Medical fournal, 1, 131.

Ellison, R. R., et al. (1968). Blood, 32, 507.

Galton, D. (1973). To be published.

Haddow, A., and Alexander, P. (1964). Lancet, 1, 452

Harris, R. (1973). Nature, 241, 95.

Mathé, G. et al. (1972). National Cancer Institute Monographs, 35, 361

Morris Jones, P. (1973). Personal communication.

Powles, R., Kay, H. E. M., et al. (1973). Procèdes de Semaine Cancerologique, 1972 de Paris. In press.

Wiernik, P. H., and Serpick, A. A. (1970). Medicine, 49, 505.

\title{
Smoking in Pregnancy and Subsequent Child Development
}

\author{
N. R. BUTLER, H. GOLDSTEIN
}

British Medical fournal, 1973, 4, 573-575

\section{Summary}

A national sample of several thousand children has been followed longitudinally from birth. At the ages of 7 and 11 years physical and mental retardation due to smoking in pregnancy has been found, and this deficit increases with the number of cigarettes smoked after the fourth month of pregnancy. Children of mothers who smoked 10 or more cigarettes a day are on average $1.0 \mathrm{~cm}$ shorter and between three and five months retarded on reading, mathematics, and general ability compared with the

Bristol Royal Hospital for Sick Children, Bristol BS2 8BJ

N. R. BUTLER, M.D., F.R.C.P., Professor of Child Health

National Children's Bureau, London EC1V 7QE

H. GOLDSTEIN, B.SC., Statistician offspring of non-smokers, after allowing for associated social and biological factors.

\section{Introduction}

It is now widely accepted that maternal smoking in pregnancy is associated with both a reduction in birth weight and an increase in perinatal mortality (Butler et al., 1972; British Medical fournal, 1973). Though there is still some dispute about the mechanism of the association the evidence from human and animal studies suggests that it is the smoking rather than the type of woman who smokes which is responsible (British Medical fournal, 1973).

The long-term effects of smoking in pregnancy on the surviving child are less well documented. Hardy and Mellits (1972) found very few significant differences in a number of body measurements and intellectual functions up to the age of 7 years between children of smokers and non-smokers. On the other hand, by using data from the National Child Development 
Study, Davie et al. (1972) and Goldstein (1972) found highly significant differences in height and reading attainment at 7 years between children of mothers who smoked and those who did not. A likely explanation for this discrepancy is that whereas Hardy and Mellits based their findings on 88 matched pairs of children, Davie et al. and Goldstein used a sample size of over 5,000 children. In the case of height, where a comparison can be made, the actual sizes of the differences found are similar in the two studies. Calculations show that with the small sample of Hardy and Mellits there was little chance (about $20 \%$ ) of detecting statistically significant differences in height (at the 5\% level) of the size found by Davie et al. and Goldstein (Donovan, 1973).

The present paper extends the results of Davie et al. and Goldstein to the most recent examination of the same children at the age of 11 .

\section{Methods}

A detailed description of the National Child Development Study can be found in Davie et al. (1972). It is a longitudinal study of the 17,000 children born in Britain from 3 to 9 March 1958. At the birth of these children the study was known as the Perinatal Mortality Survey of the National Birthday Trust Fund, and at the two follow-ups at the ages of 7 and 11 years as the National Child Development Study of the National Children's Bureau.

Comprehensive information about birth weight, gestation, and maternal social and obstetric characteristics was obtained at birth, as well as information about smoking habits during pregnancy. The test procedures included a test of reading at the age of 7 , and a mathematics test, a reading test, and a general ability test at the age of 11 . At both ages the height of the child was also measured. It was shown by Butler et al. (1972) that the effect of smoking on raising the perinatal mortality rate and on lowering birth weight was determined by smoking habits after the fourth month of pregnancy. Thus, for example, if the mother had given up smoking by the fourth month then the mortality risk and expected birth weight were the same as for mothers who had not been smokers before pregnancy. The analyses at 7 and 11 years are therefore based on smoking habits after the fourth month of pregnancy.

\section{Results}

Statistically significant differences in height and reading ability between smoking categories were found at the age of 7 .

To allow for the possibility that these observed relationships are mediated by those characteristics which measure the kind of woman who smokes and who also has shorter and less able children, the following factors were taken into account in a multifactorial analysis: mother's height, age, social class as determined by the father's occupation, number of older and number of younger children in the household, and the sex of the child. Full details are given elsewhere (Davie et al., 1972).

When account was taken of all these factors there was a deficit of height and reading ability in the offspring of mothers who smoked, the extent of which increased with the amount smoked. The average difference between children of mothers who smoked 10 or more cigarettes a day and those of nonsmokers was $1.0 \mathrm{~cm}$ in height and four months in reading attainment. By comparison with the effects of some of the other factors such as social class and the number of older and younger children in the household, the effect of smoking in pregnancy is relatively small. For example, the difference between having three or more older children in the child's household compared with having none is $2.0 \mathrm{~cm}$ in height and 15 months in reading attainment.

The 7-year-old analyses were repeated after the children had been studied at 11, this time with four "outcomes": a general ability test, a reading comprehension test, a mathematics test,

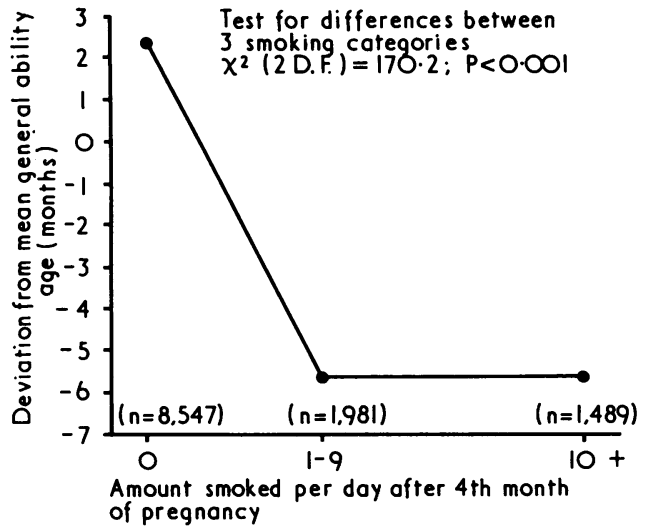

FIG. 1-General ability of 11-year-old children whose mothers smoked after fourth month of pregnancy.

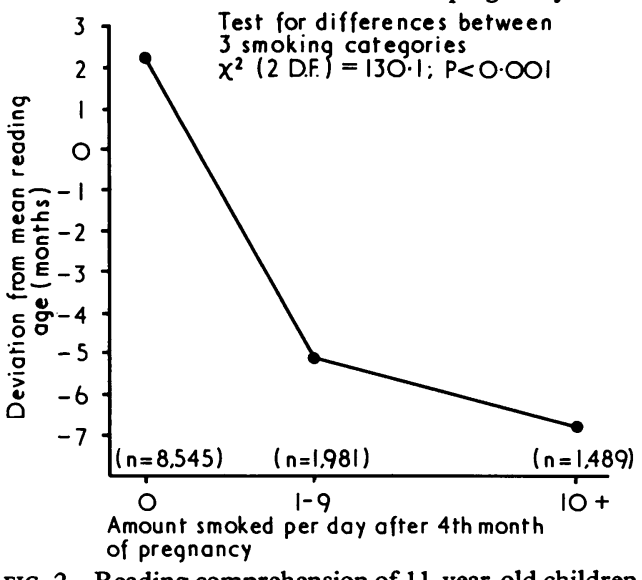

FIG. 2-Reading comprehension of 11-year-old children whose mothers smoked after fourth month of pregnancy.

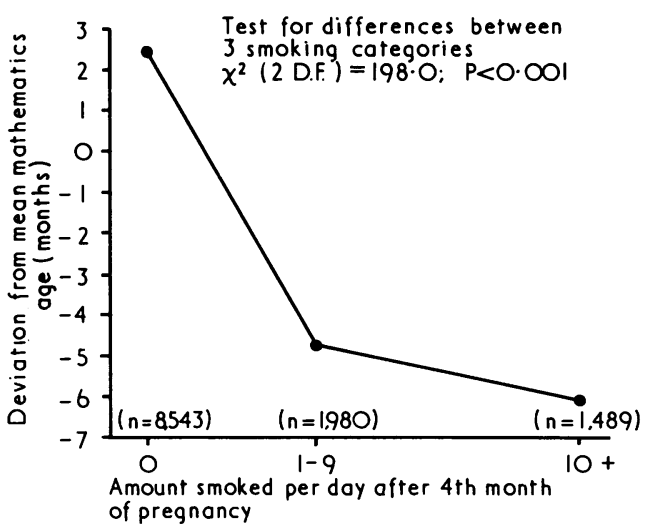

FIG. 3-Mathematics ability of 11-year-old children whose mothers smoked after fourth month of pregnancy.

and height. The mean scores for the three tests and height by smoking categories are shown in figs. 1 to 4 . After adjusting for the same associated factors as at the age of 7 a similar pattern is shown in figs. 5 to 8 , where differences persist but are reduced. The average difference between children of women who smoked 10 or more cigarettes a day and those who smoked none was three months for general ability (eight months before adjustment) four months for reading (nine months before adjustment), five months for mathematics (eight months before adjustment), and $1.0 \mathrm{~cm}$ for height $(1.6 \mathrm{~cm}$ before adjustment).* For the three

*Tests of significance for differences between the three smoking categories (none, 1-9, 10 or more) were carried out after fitting constants for the other factors. The $\chi^{2}$ test statistic was used rather than the $F$ test since the denomifactors. The $\chi^{2}$ test statistic was used rather than the $F$ test since the denominator degrees of freedom are large. The numbers of cases in each analysis
fluctuate since not all the children in the study had complete information. fluctuate since not all the children in the study had complete information.
In particular, the numbers entering into the analyses which adjusted for In particular, the numbers entering into the analyses which adjusted for other factors are considerably reduced. However, a study of the cases with
incomplete data does not suggest that any serious bias has resulted from their exclusion. 


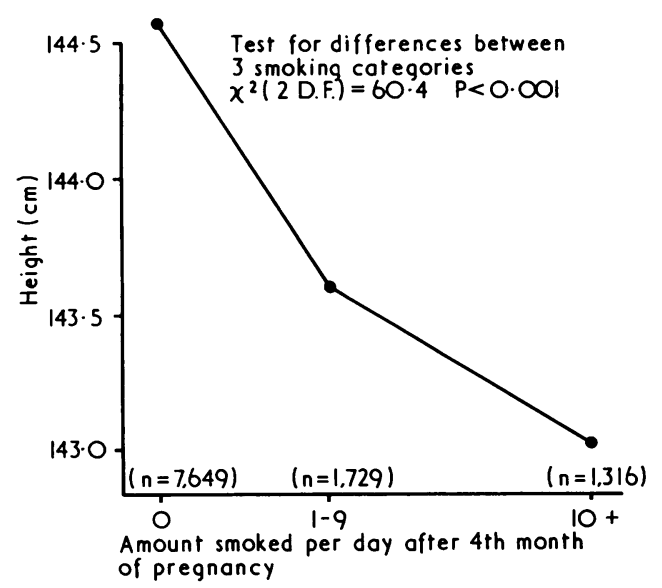

FIG. 4-Height of 11-year-old children whose mothers smoked after fourth month of pragnancy.

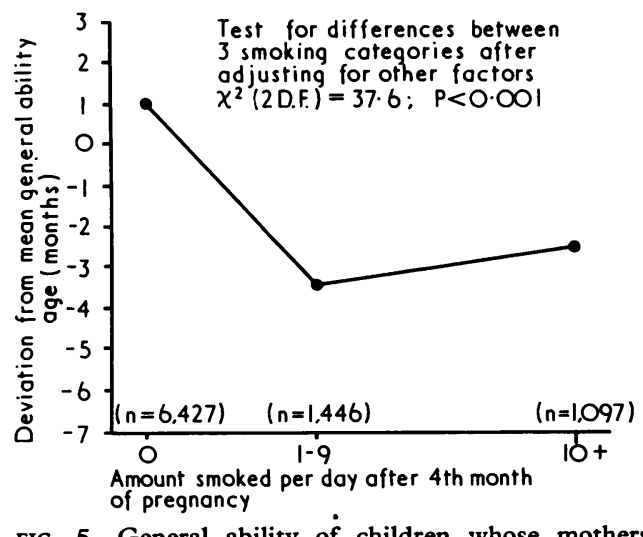

FIG. 5-General ability of children whose mothers smoked after fourth month of pregnancy, after adjusting for other factors (see text).

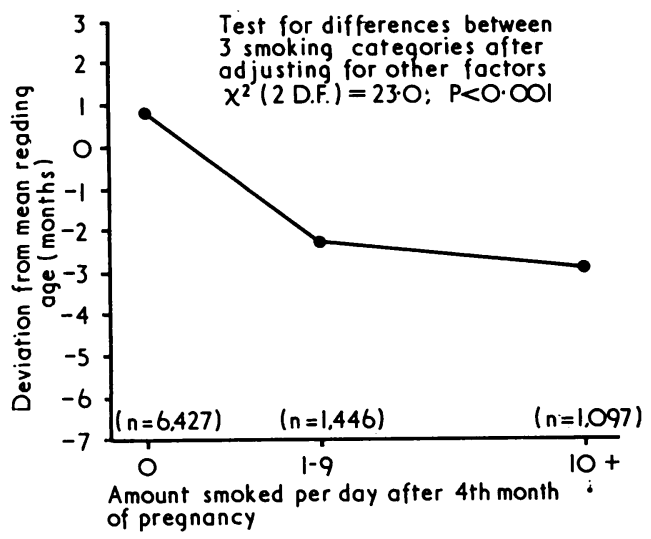

FIG. 6-Reading comprehension of 11-year-old children whose mothers smoked after fourth month of pregnancy, after adjusting for other factors.

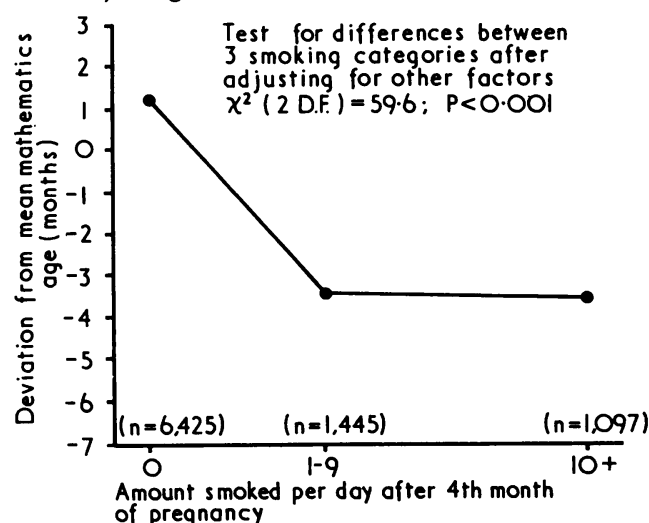

FIG. 7-Mathematics ability of 11-year-old children whose mothers smoked after fourth month of pregnancy, after adjusting for other factors.

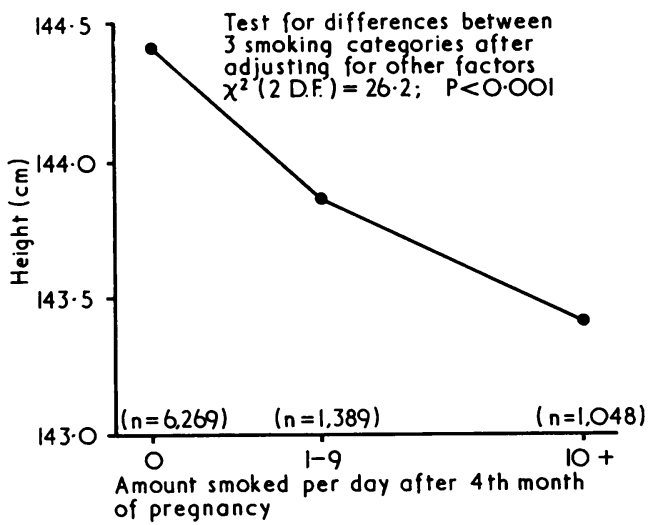

FIG. 8-Height of 11-year-old children whose mothers smoked after fourth month of pregnancy, after adjusting for other factors.

test scores, though not for height, there appears to be little decrease in score beyond the category of 1-9 cigarettes smoked per day.

As at 7 years, the differences between smokers and nonsmokers are less than the effects of some of the other factors. For example, the difference between a child from a household with no older children and one from a household with three or more is 16 months for general ability, 29 months for reading, 14 months for mathematics, and $4.0 \mathrm{~cm}$ for height.

\section{Discussion}

These results establish a continuing effect of smoking in pregnancy, though the gap between children of smokers and nonsmokers (at all levels of smoking) has not widened between the ages of 7 and 11.

Smoking in pregnancy is associated with an impairment of both mental and physical growth, though compared with the other sociobiological factors used in the present analysis the effects are small.

The present results fail to support the hypothesis that it is entirely the type of mother rather than the smoking which is causative, in so far as a mother can be typified by those factors which were selected for analysis. The analysis has shown, however, that these factors do account for some part of the association. In spite of the fact that not every possibly relevant influence has been allowed for-for example, personality factors-these results may be seen as lending support to the hypothesis that it is the smoking itself which, at least in part, "causes" the impairment. Moreover, if it is accepted that the relation with birth weight and perinatal mortality is "causal" it would seem reasonable to accept a similar conclusion regarding the longer-term effects of maternal smoking on child development.

We would like to thank the following for their helpful comments: Mrs. E. Ferri, Mr. K. Fogelman, Mr. M. J. R. Healy, Dr. J. Parfit, Dr. M. L. Kellmer Pringle, Dr E. Ross, and Dr. M. Sheridan.

This work was partly supported by grants to the National Children's Bureau from the Social Science Research Council and the Department of Health and Social Security.

\section{References}

British Medical fournal, 1973, 1, 369.

Butler, N. R., Goldstein, H., and Ross, E. M. (1972). British Medical fournal, 2, 127.

Davie, R., Butler, N. R., and Goldstein, H. (1972). From Birth to Seven. London, Longmans

Donovan, J. (1973). Lancet, 1, 376

Goldstein, H. (1972). Human Biology, 43, 92.

Hardy, J. B., and Mellits, D. D. (1972). Lancet, 2, 1332. 